[Aus dem hygienischen Institut in Heidelberg.]

(Direktor: Prof. Dr. H. Kossel.)

\title{
Über die Desinfektion von Tierhaaren zur Verhütung von gewerblichem Milzbrand. ${ }^{1}$ \\ Von
}

Dr. K. Laubenheimer.

Privatdozenten und I. Assistenten.

Unter den übertragbaren Krankheiten, welche bestimmte Gewerbe besonders gefährden, nimmt an Bedeutung der Milzbrand die erste Stelle ein. Da der Milzbrand beim Menschen fast ausschlieBlich durch Übertragung der Krankheitskeime von Tieren oder tierischem Material zustande kommt, so sind solche Personen, die mit der Pflege von Tieren oder mit der Verarbeitung tierischer Produkte beschäftigt sind, in erster Linie einer Infektion ausgesetzt. Die gröBte Zahl der Erkrankungen findet sich bei Schlächtern, an zweiter Stelle folgen Personen, die in Fabriken beschäftigt sind, in welchen von Tieren stammendes Material verarbeitet wird, so vor allen in Gerbereien, RoBhaarspinnereien, Bürsten- und Pinselfabriken. Auch bei Woll- und Lumpensortierern, bei Pelz- und Handschuharbeitern, Sattlern und Schuhmachern kommt eine Infektion mit Milzbrand hier und da dureh infiziertes Material zustande.

Im Gegensatz zum Milzbrand beim Tier, wobei stomachale Infektion die Regel bildet, entwickelt sich die Krankheit beim Menschen in der übergroBen Mebrzahl der Fälle als Impfmilzbrand in Form der Pustula maligna, verursacht durch das Eindringen der Krankheitskeime in kleine Hautverletzungen, Kratzwunden u. dgl.

1 Nach einem Vortrag, gehalten auf der 83. Versammlung deutscher Naturforscher und Ärzte in Karlsruhe. 1911.

Zeitschr. f. Hygiene. IXX 
Weniger häufig findet sich beim Menschen Lungenmilzbrand, hervorgerufen durch Aufnahme der Keime mit der Atemluft. Diese zuerst in England in gröBerem Umfange beobachtete und unter dem Namen der „Woolsorters disease" bekannt gewordene Form des Milzbrandes findet sich hauptsächlich bei Lumpensortierern und Wollarbeitern.

Noch seltener erfolgt eine direkte Infektion des Darmes beim Menschen, die unter Umständen dann zustande kommt, wenn Nilzbrandsporen in die Nahrung durch infizierte Hände oder auf andere Weise hineingelangen.

Wenn nun auch in Deutschland dank der guten veterinärpolizeilichen Gesetzgebung der Milzbrand unter den Tieren bei weitem nicht' so verbreitet ist, wie in anderen Staaten, und demgemäB auch die Infektionen beim Menschen nicht so häufig sind, wie dort, so ist dennoch die Zahl der alljährlich im Deutschen Reiche an Milzbrand Erkrankten und Gestorbenen eine recht beträchtliche.

So erkrankten z. B. in Preußen in den Jahren 1905 bis 1910 einschlieBlich 868 Personen an Milzbrand, von denen $117=13.5$ Prozent der Krankheit erlagen.

Solche Zahlen zeigen, daß die Gewerbehygiene allen Grund hat, nach Mitteln und Wegen zu suchen, um die Gefahr der Infertion in den genannten Betrieben zu verringern. Auch die gesetzgebenden Körperschaften haben sich dieser Ansicht nicht verschlossen und eine Reihe Bestimmungen in diesem Sinne erlassen.

So dürfen nach dem Reichsgesetz, betreffend Abwehr und Unterdrūctiung von Viehseuchen, Kadaver der gefallenen oder getöteten milzbrandkranken oder der Seuche verdächtigen Tiere nicht abgehäutet werden. Nach dem Reichsgesetz, betreffend die Bekämpfung übertragbarer Krankheiten vom 28. September 1909, ist ferner der Milzbrand beim Menschen anzeigepflichtig. SchlieBlich sind für die einzelnen Betriebe, die sich mit der Verarbeitung von Tieren stammenden Materials befassen, besondere gesetzliche Bestimmnngen erlassen, so die Bekanntmachung des Reichskanzlers, betreffend die Einrichtung und den Betrieb der RoBharspinnereien, Haar- und Borstenzurichtereien, sowie der Bürsten- und Pinselmachereien vom 22. Oktober 1902, wonach aus dem Auslande stammende Pferde- oder Rinderhaare, Schweinsborsten und Schweinswolle, sowie Ziegenhaare desinfektionspllichtig sind. In dem ursprünglichen ErlaB von 1899 waren Ziegenhaare nicht unter dem desinfektionspflichtigen Material aufgenommen. Erst auf Grund eines Milzbrandfalles beim Menschen durch Ziegenhaare und des gelungenen Nachweises der Infektionserreger an diesem Material durch Heim (1), müssen auch ausländische Ziegenhaare einer Desinfektion vor ihrer Verarbeitung unterzogen werden. 
Wie wichtig und nötig diese Bestimmung ist, werde ich noch auszuführen haben.

Die Gelegenheit zur Infektion in Roßhaarspinnereien ist während der Verarbeitung der Haare in reichem MaBe gegeben. Die Haare werden zunächst nach der Farbe mit der Hand sortiert, was natürlich nicht ohne groBe Staubentwicklung vor sich geht. Dann werden die Haare ,zerrissen", wodurch die Haarbündel in die einzelnen Haare zerlegt werden. Hierzu dienen Maschinen, der sogenannte "Reißwolf", eine sich drehende, mit Stacheln besetzte Walze. Zuletzt gelangen die Haare in den "Mischwolf". In beiden Maschinen ist die Staubentwicklung eine ganz gewaltige, und trotz Schutzmantel und Absaugevorrichtung ist es nicht ganz zu vermeiden, daB Staub in den Arbeitsraum dringt.

Auch in Haar- und Borstenzurichtereien, sowie in Bürsten- und Pinselmachereien sind die Arbeiter Milzbrandinfektionen ausgesetzt, da auch in diesen Betrieben Haare milzbrandempfänglicher Tiere, wie von Pferden und Schweinen, verarbeitet werden. So sind denn auch Milzbrandfälle in den genannten Fabriken wiederholt beobachtet worden und das Rohmaterial, sobald es aus dem Ausland bezogen wird, ist desinfektionspflichtig. Nicht dagegen ist bis jetzt die Desinfektion von Wolle vorgeschrieben, trotzdem das Verarbeiten von Wolle ebenfalls, wie erwähnt, mit Milzbrandgefahr verknüpft ist. In den letzten Jahren sind mehrere Infektionen mit zum Teil tödlichem Ausgang in einer Wollkämmerei vorgekommen, wie ich einer Arbeit von Weller (2) in der ,Vierteljahrsschrift für gerichtliche Medizin" entnehme.

Während man nun bisher kein wirksames Verfahren kennt, um Tierhäute sicher zu desinfizieren, ohne das Material für die Verwendung als Leder oder Kürschnerware unbrauchbar zu machen, hat man, wenn es sich nur um die Desinfektion von Haaren handelt, mehrere Kethoden, von denen drei in dem schon erwähnten Gesetz vorgesehen sind:

1. Desinfeltion des Rohmaterials durch 30 Minuten dauernde Einwirkung strömenden Wasseràampfes bei 0.15 Atmosphären Überdruck.

2. 15 Minuten langes Kochen in $\mathbf{2}$ prozent. Kaliumpermanganatlösung, Bleichen in 3 prozent. schwefliger Säure und Trocknen an der Sonne.

\section{2 stündiges Kochen.}

Trotzdem jedes der drei Verfahren geeignet ist, Mrilzbrandkeime abzutöten, hat die Zahl der Erkranknngen an Milzbrand in den Betrieben, welche Tierhaare verarbeiten, eher zu- als abgenommen. Aus diesem Grunde sah sich das badische Ministerium des Innern veranlaBt, eine behördliche Überwachung der gesetzlichen Desinfektionsmaßnahmen in den betreffenden Fabriken anzuordnen, wonach die zuständigen Bezirks- 
ärzte angewiesen sind, einmal jährlich in den Fabriken ihres Bezirks frisch desinfizierte Haarproben unter aseptischen Kautelen zu entnehmen und den Untersuchungsämtern für Infektionskrankheiten in Freiburg oder Heidelberg zur bakteriologischen Untersuchung einzusenden. Diese Bestimmung ist seit dem 28. Oktober 1909 in Kraft.

Über die Ergebnisse dieser Untersuchungen und über die Erfahrungen, die dabei in dem Untersuchungsamte des Hygienischen Institutes in Heidelberg gesammelt werden konnten, soll in folgendem kurz berichtet werden.

Die bakteriologische Prüfung der eingesandten Haarproben erwies zunächst, daß keines der vorhin erwähnten drei Desinfektionsverfahren imstande ist, alle den Haaren anhaftenden Keime zu vernichten. Die besten Ergebnisse in dieser Hinsicht lieferte das chemische Bleichverfahren, d. h. das Kochen mit 2 prozent. Kaliumpermanganat, Bleichen in 3 prozent. schwefliger Säure und Trocknen in der Sonne, wobei durch das Kochen in der Kaliumpermanganatlösung auch eine weitgehende mechanische Entfernung anhaftender Keime von den Haaren anzunehmen ist.

Weniger gute Resultate erzielte die Desinfektion mit gespanntem Dampf, wirkte aber immer noch energischer keimtōtend, wie das 2stündige Kochen.

Was die Art der Keime betrifft, die diesen energischen Desinfektionsverfahren standhalten können, so handelt es sich natürlich nur um solche Arten, die sehr widerstandsfähige Dauerformen bilden, wie die Heu-, Kartoffel- und Erdbazillen, die sich stets in großen Mengen an den Haaren nachweisen lassen.

Mitunter fanden sich auch vegetative Keime an desinfizierten Haaren, so Bact. coli und Staphylokokken, ein Befund, auf dessen Bewertung ich noch zu sprechen kommen werde.

Dreimal wurde die Untersuchung von Haarproben verlangt im AnschluB an Milzbrandinfektionen, die sich in den betreffenden Fabriken ereignet hatten. In allen Fällen gelang es, die Ursache der Erkrankungen in Form von Milzbrandsporen an dem verdächtigen Material nachzuweisen.

Die Zahl der bisher veröffentlichten Fälle, in denen die vermuteten Krankheitserreger an dem verdächtigen Material durch bakteriologische Methoden nachgewiesen werden konnten, ist gering. In der deutschen Literatur finden sich auBer dem erwähnten Falle von Heim bis zu diesem Jahre nur fünf derartige Angaben (3). Einige weitere positive Befunde sind in England erhoben und in einer sehr ausführlichen Abhandlung über „British Industrial Anthrax" von Page (4) zusammengestellt. Erst auf der diesjährigen fünften Tagung der Freien Vereinigung für Mikrobiologie in Dresden berichtete Reichel (5) über zehn voneinander unabhängige 
Einzelfälle von Milzbrandinfektionen in Gewerbebetrieben, in denen neunmal die Krankheitserreger auf dem verarbeiteten tierischen Material nachzuweisen waren. In sieben Fällen handelte es sich um Übertragung der Keime durch Tierhaare, wobei sechsmal Milzbrandsporen in der AuBenwelt aufgefunden werden konnten, und zwar erwiesen sich durchweg RoBhaare als Träger der Infektion. Aus diesen Befunden schlieBt Reichel entweder auf ein weitverbreitetes Vorkommen von Milzbrandsporen auf tierischen Rohstoffen, oder auf eine besonders leichte Feststellbarkeit der Infektionsquelle. Aus den Mitteilungen des genannten Autors ist noch hervorzuheben, daB der Nachweis der Milzbrandkeime nur an solchen Proben gelang, die nachweislich oder wahrscheinlich nicht vorschriftsmäBig desinfiziert waren.

In unserem ersten positiven Fall handelte es sich um eine RoBhaarprobe, die $\mathrm{n}$ a ch der vorschriftsmäBigen Desinfektion von dem Bezirksarzt entnommen und dem Untersuchungsamt eingeschickt war, nachdem kurz zuvor sich ein Arbeiter in der betreffenden Fabrik eine tödlich verlaufende Milzbrandinfektion zugezogen hatte. Es gelang, an den zugesandten Haaren Milzbrandkeime nachzuweisen, die also durch die Desinfektion im Dampfapparat nicht abgetötet worden waren.

Der Grund für die Unwirksamkeit der Desinfektion war in einer fehlerhaften Konstruktion des Desinfektionsapparates oder in einem Fehler in der Bedienung desselben zu suchen.

Mit Genehmigung des Ministeriums des Innern wurde deshalb die Haardesinfektion in der betreffenden Fabrik einer Revision unterzogen. Es fand sich dabei nichts, das zur Beanstandung hätte führen können. Zur Desinfektion kam ein Ballen russischer Pferdeschweifhaare nach vorheriger Lockerung der zusammenhaltenden Schnüre. In verschiedenen Tiefen des Ballens wurden Maximalthermometer, ein Klingelthermometer sowie Testobjekte, bestehend aus Milzbrandsporenfäden, untergebracht. Vor Beginn der Desinfektion und nach Beendigung derselben. wurden Haarproben aus verschiedenen Teilen des Ballens mit sterilen Instrumenten entnommen und in sterile Gläser gebracht, die ausgelegten Testobjekte nach der Desinfektion gesammelt. Die im Hygienischen Institut rorgenommene Untersuchung der entnommenen Proben ergab, daß den desinfizierten Haaren nur sporenbildende Keime aus der Gruppe der Heu- und Erdbazillen anhafteten. Milzbrandkeime ließen sich nicht nachweisen. Die Milzbrandsporenfäden waren steril. Aus diesem Befund mußte geschlossen werden, daB die ungenügende Desinfektion, die zu der erwähnten Milzbrandinfektion geführt hatte, nicht dureh einen Mangel des Desinfektionsapparates, sondern durch einen Fehler in der Bedienung oder 
in einer nicht genügenden Auflockerung des betreffenden Haarballens bedingt war.

Um Anhaltspunkte über die Widerstandsfähigkeit der den Haaren anhaftenden Sporen zu gewinnen, wurden die in der Fabrik desinfizierten Haare im Koch schen Dampftopf nochmals $1 / 2$ und 1 Stunde strömendem Dampf von $100^{\circ}$ und eine weitere Probe dieselbe Zeit Wasserdampf von $105^{\circ}$ im Autoklaven ausgesetzt. Auch jetzt noch ließen sich an den so behandelten Haaren entwicklungsfähige Sporen nachweisen.

Diese Versuche zeigen, daß eine vollständige Sterilisation der Tierhaare praktisch nicht zu erreichen ist. Wenn es schon bei den kleinen, zu den erwähnten Versuchen benutzten Proben, wobei der Dampf ungehinderten Zutritt zu den Haaren hatte, nicht möglich war, die resistenten Dauerformen abzutöten, so ist das noch viel weniger zu erwarten bei den voluminösen Haarballen, wie sie in der Fabrik zur Desinfektion kommen. Auf Grund dieser Erfahrungen schließen wir bei den eingesandten Haarproben dann auf eine ausreichende Desinfektion, wenn keine Milzbrandkeime nachgewiesen werden können, und wenn ferner keine Kolonien regetativer Bakterienarten in der Kultur zur Entwicklung kommen. Das Auskeimen sporentragender Arten aus der Gruppe der Heu-, Erd- und Kartoffelbazillen berechtigt nicht zu dem Schlusse, daB die Desinfelition eine ungenügende war.

Der Nachweis von Milzbrandkeimen an Tierhaaren war bisher stets nur an Pferdehaaren erhoben worden, ausgenommen der eine schon erwähnte Fall von Heim, der dazu Veranlassung gab, daB auch ausländische Ziegenhaare unter das desinfeltionspllichtige Material aufgenommen wurden. Wie richtig und nötig diese Bestimmung ist, kann ich durch eigene Erfahrungen bestätigen, wonach vier Milzbranderkrankungen mit einem Todesfall auf eine Infektion durch Ziegenhaare zurückzuführen sind.

Bei den gesteigerten Preisen werden die geringwertigen RoBhaarsorten in ausgedehntem MaBe mit den billigeren Ziegenhaaren vermischt, und es ist nicht unwahrscheinlich, daB mit auf diesen Umstand die in letzter Zeit auffallende Vermehrung von Milzbranderkrankungen in RoBhaarspinnereien zurückzuführen ist. Die zur Verarbeitung kommenden Ziegenhaare werden fast stets aus dem Ausland bezogen, aus China, Indien, Afrika, Südamerika, Rußland und Spanien. Seltener werden sogenannte Gerbereihaare verwendet, die in deutschen Gerbereien bei dem Enthaarungsprozeß gewonnen werden.

DaB Ziegenhaare in bezug auf Milzbrandgefahr ganz besonders gefährliche Objekte darstellen, geht aus unseren Untersuchungen hervor, wonach dieselben unter Umständen hochgradig mit Milzbrandsporen infiziert sein können und auBerdem einer sicheren Sterilisation noch gröBere 
Schwierigkeiten entgegensetzen, wie Pferdehaare. Ich werde auf diesen Punkt noch zurückkommen.

Die ersten von uns beobachteten Fälle von Milzbrandinfektion durch Ziegenhaare ereigneten sich in einer RoBharspinnerei in Mannheim, unter deren Arbeitern fast gleichzeitig drei Fälle von Hautmilzbrand auftraten, von denen der eine rasch tödlich verlief. Die Erkrankten waren alle in der Hechelei beschäftigt gewesen und nur mit Material in Berührung gekommen, das den Desinfektionsapparat durchlaufen hatte.

Als Träger der Infektion kamen vornehmlich indische Ziegenhaare in Betracht, mit deren Verarbeitung die Erkrankten in tun hatten.

Von dem verdächtigen Material wurden verschiedene Proben vor der Desinfektion entnommen und schließlich ein weiterer Ballen Ziegenhaare der Desinfektion unterworfen, nachdem Maximal- und Klingelthermometer sowie Milzbrandsporenfäden als Testobjekte an verschiedenen Stellen des Apparates und in verschiedenen Schichten des Ballens untergebracht waren.

Vor der Desinfektion wurden die Eisenbänder, die den etwa 3 Zentner schweren, hydraulisch gepreBten Ballen zusammenhielten, entfernt, wobei sich zeigte, daB die Haarbündel nur sehr geringe Neigung hatten, auseinanderzugehen, während RoBhaarballen nach Entfernung der Umschnürung infolge der großen Elastizität der RoBhaare leicht in einzelne Bündel zerfallen. Die Ziegenhaarballen fühlten sich fast steinhart an, und um die Testobjekte und Thermometer in tieferen Schichten unterzubringen, muBte mit Hammer und Stemmeisen ein Loch in die verfilzten Haarmassen geschlagen werden.

Unter solchen Umständen war es njcht zu verwundern, daß nach $1 / 2$ stündiger Einwirkung des Dampfes mit 0.5 Atmosphären Überdruck $20^{\mathrm{cm}}$ unter der Oberfläche des Ballens erst eine Temperatur von $46^{\circ}$ erreicht war, eine Desinfektion mithin nicht stattgefunden haben kounte.

Die bakteriologische Untersuchung der entnommenen Proben hatte nachstehendes Resultat. An den Haaren eines nicht desinfizięrten Ballens von der Oberfläche und aus den tiefen Schichten fanden sich neben zahlreichen vegetativen und sporenbildenden Keimen echte Milzbrandbazillen.

Haare von der Oberfläche eines weiteren Ballens nach der Desinfektion wiesen nur wenige Keime aus der Gruppe der Heu- und Erdbazillen auf. Milzbrandsporen wurden in dieser Probe nicht gefunden. Dagegen konnten Milzbrandkeime wieder nachgewiesen werden an Proben, die aus Schichten ungefähr $20^{\mathrm{cm}}$ unter der Oberfläche stammten.

Kurz zusammengefaBt hatte also die beschriebene Untersuchung, die sich an drei Fälle von Milzbrand bei Arbeitern anschloB, die mit desinfizierten Ziegenhaaren $\mathrm{zu}$ tun hatten, ergeben, daB das verarbeitete Material hochgradig mit Milzbrandsporen infiziert war. Ferner, daB in- 
folge der geringen Elastiaitat der Ziegenhare eine wirksame Desinfektion anf grobe Schwierigkeiten stöBt und sich nur auf die oberfächlichen Schichten der Haarballen beschränkt.

$21 / 2$ Monate später ereignete sich in Lahr in einer RoBhaarspinnerei ein Fall ron Hautmilzbrand, als dessen Infektionsquelle ebenfalls Ziegenhaare in Betracht kamen. Die daraufhin vorgenommene Dntersuchung des der Infektion verdächtigen Materials ergab wieder Milzbrandbazillen an den Ziegenhaaren, die aber diesmal nur an nicht desinfizierten Proben vorgefunden wurden. An den desinfizierten Haaren konnten nur Heuund Erdbazillen nachgewiesen werden. Vielleicht waren die eingesandten Haarproben nur obertlächlichen Schichten entnommen, bis zu welchen der Dampf eine genügende Wirkung entfalten konnte. Die mit Milzbrandsporen infizierten Ziegenhaarballen stammten von derselben Schiffsladung, wie die Ballen, welche die Infektionen in Mannheim verursacht hatten. Es muB aus dem Umstand, daB bei drei Stichproben sich regelmäBig Milzbrandsporen nachweisen ließen, geschlossen werden, daß mit grober Wahrscheinlichleit die ganze Schiffsladung infiziert war.

Ich gehe nunmehr zu den bakteriologischen Methoden ūber, welche den Nachweis von Milzbrandkeimen an Tierbaaren gestatten. Ich erwähnte schon die aufällige Tatsache, daB ein positiver Befund bisher in so wenigen Fällen erhoben worden ist, wäbrend nach den Untersuchungen von Reichel und nach unsecen Erfahrungen der Nachweis dureh das Kulturverfabren keine besonderen Schwierigkeiten bietet, falls überbaupt Milzbrandkeime an dem Material vermutet werden dürfen.

Eine Anreicherungsmethode anzuwenden, so durch Jैbergießen der Haare mit Bouillon und Bebrütung bei $37^{\circ}$, empfieblt sich nicht, da die Begleitbakterien etwa vorhandene Milzbrandkeime vollstāndig überwuchern, auch wenn man durch Erhitzen auf 60 bis $80^{\circ}$ für die Entfernung der vegetativen Formen Sorge getragen hat. So erhielt Heim in dem erwähnten Falle eines gelungenen Nachweises von Milzbrandsporen an Ziegenhaaren mit einer solchen Anreicherung nur negatire Resultate, während der Nachweis der Infektionserreger gelang, als er, einem Vorschlage Kossels folgend, die Haare mit Bouillon abschüttelte, die vegetativen Formen durch Erhitzen auf $80^{\circ}$ abtötete, sofort zentrifugierte und den Bodensatz auf Agarplatten ausstrich. Nach dem gleichen Prinzip arbeitend, erhielt Reichel seine positiven Befunde. Reichel übergieBt die Proben mit sterilem Wasser, erhitzt $3 / 4$ Stunden auf $65^{0}$, zentrifugiert und benutzt den Bodensatz zur Aussaat auf Agar in groBen Schalen.

Noch einfacher gestaltet sich die Methodil, die uns die Infektionskeime an Tierhaaren mit RegelmäBigheit nachzuweisen gestattete. Es 
wurde dabei sowohl auf ein Abschütteln der Keime von den Haaren durch Bouillon oder Wasser, sowie auch auf das Erhitzen zwecks Abtötung der vegetativen Bakterien verzichtet, was um so eher geschehen konnte, als wir es meistens mit Proben zu tun hatten, die in der Fabrik schon desinfiziert waren und deshalb keine oder nur wenige vegetative Formen aufwiesen. Ist dagegen das Material, wie immer in undesinfiziertem $\mathrm{Zu}$ stande, sehr mit solchen Bakterien verunreinigt, so wird durch das Erhitzen die Untersuchung sicher erleichtert. Das von uns geübte Verfahren bestand demnach darin, daB von den zu untersuchenden Haarproben etwa $1 \mathrm{~cm}$ lange Stücke mit sterilen Instrumenten abgeschnitten, in verschiedenen Mengen in Petrischalen gegeben und mit etwa $60^{\circ}$ heißem Agar übergossen wurden. Durch Hin- und Herneigen der Schalen werden die Haare in dem Nährmedium gut verteilt. Nach 16 bis 20 Stunden bei $37^{\circ}$ gelangen die gewachsenen Kolonien zur Untersuchung. Die Unterscheidung milzbrandähnlicher von echten Milzbrandkolonien nur nach der Wachstumsform kann auch dem. geübten Untersucher Schwierigkeiten bereiten. Von den verdächtigen Kolonien werden deshalb mit verdünntem Karbolfuchsin und nach Gram gefärbte Ausstrichprāparate sowie hängende Tropfen angefertigt. Namentlich die Untersuchung im hängenden Tropfen gibt sehr wertvolle Anhaltspunkte, da die Mehrzahl der milzbrandähnlich wachsenden Bakterienarten im Gegensatz zu den echten Milzbrandbazillen Eigenbewegung besitzt. Zeigt sich eine Kolonie, die der Form nach auf Milzbrand verdächtig ist, aus grampositiven unbeweglichen Stäbchen zusammengesetzt, so wird davon auf Schrägagar und Bouillon abgeimpft sowie gleichzeitig eine Maus oder ein Meerschweinchen damit subkutan geimpft. In der Mehrzahl der Fälle impften wir nur Mäuse mit verdächtigen Kolonien und erhielten dabei stets rasch tödlich verlaufende Infektionen, ein Umstand, der deshalb hervorgehoben sei, weil andere Autoren (Petruschky) ${ }^{1}$ bei der Verwendung von Meerschweinchen zuweilen positive, mit Mäusen dagegen negative Resultate erhalten haben. Der Ausfall des Tierversuchs ist entscheidend für die Diagnose und gibt, wenn Milzbrand vorliegt, meist schon innerhalb 24 bis 48 Stunden das endgültige Resultat.

Wenn nun auch der Tierversuch zur Identifizierung verdächtiger Kolonien unentbehrlich ist, so versagt er sehr häufig bei direktem Verimpfen milzbrandverdächtigen Materials, selbst wenn dasselbe im Kulturverfahren sich als infiziert erweist, das also in diesem Falle das feinere Reagens darstellt.

${ }^{1}$ Diskassionsbemerkung auf der 83. Versammlung deutscher Naturforscher und Ärzte. Karlsruhe 1911. 
Um das Gesagte noch einmal kurz zusammenzufassen, so sehen wir, daB in den Gewerben, die sich mit der Verarbeitung von Tieren stammenden Materials beschäftigen, zahlreiche Erkrankungen und Todesfälle an Milzbrand alljährlich beobachtet werden, die auf Infektionen durch das verarbeitete Material zurückzuführen sind. In hervorragendem MaBe sind die Arbeiter in Betrieben gefährdet, in welchen Tierhaare verarbeitet werden, also in RoBhaarspinnereien, Borsten- und Pinselfabriken. Als besonders infektionsgefährlich sind die aus dem Auslande bezogenen Tierhaare anzusehen, die aus diesem Grunde vor der Verarbeitung einer Desinfektion zu unterwerfen sind. Trotz dieser gesetzlich vorgeschriebenen VorsichtsmaBregel kommen Milzbrandfälle unter den Arbeitern der genannten Betriebe nicht selten vor. So ereigneten sich in dem Teil Badens, für den das Untersuchungsamt des Hygienischen Institutes in Heidelberg zuständig ist, in den letzten $2 \mathrm{Jahren}$ fünf Fälle von Milzbrandinfektionen in RoBhaarspinnereien, von denen zwei tödlich verliefen. Die Erkrankten hatten ausschlieBlich mit Material zu tun gehabt, das der vorschriftsmäBigen Dampfdesinfektion unterworfen war.

In dem ersten Falle konnten RoBhaare, die in der Fabrik vorschriftsmäBig desinfiziert waren, als Infelitionsträger ermittelt werden, indem lebende Milzbrandkeime an den Haaren nachgewiesen wurden.

In den vier anderen Fällen kamen als Infektionsquelle in erster Linie indische Ziegenhaare in Betracht. Die bakteriologische Untersuchung dieser Haare zeigte dieselben als mit Milzbrandsporen hochgradig. infiziert.

Welche SchluBfolgerungen und Lehren lassen sich aus diesen Untersuchungsergebnissen ziehen?

Zunächst zeigte es sich, daß die vorgeschriebene Dampfdesinfektion der ausländischen RoBhaare mitunter nicht imstande ist, anhaftende Milzbrandsporen abzutöten. Ein Fehler in der Konstruktion des Desinfektionsapparates oder eine mangelhafte Bedienung braucht dabei nicht vorzuliegen. Ein MiBerfolg kann vielmehr auch in der Beschaffenheit des Desinfektionsgutes (nicht genügende Lockerung der Ballen) seine Ursache haben.

Ferner haben unsere Untersuchungen dargetan, daB auch Ziegenhaare, die als Ersatz für RoBhaare in immer ausgedehnterem Maße verarbeitet werden, unter Umständen hochgradig mit Milzbrandkeimen infiziert sein können. Die Desinfektion der Ziegenhaarballen stöBt aber auf noch gröBere Schwierigkeiten, wie die der RoBhaarballen, da die Ziegenhaare weit weniger elastisch als RoBhaare sind und in gepreBtem Zustande, in dem sie zum Versand kommen, dem Eindringen des Dampfes weit größere Schwierigkeiten entgegenstellen. 
Nach diesen Erfahrungen ist es notwendig, die Haarballen vor dem Einbringen in den Desinfektionsapparat nicht nur von den zusammenhaltenden Umschnürungen zu befreien und hierdurch eine gewisse Auflockerung herbeizuführen, sondern die Ballen müssen vollständig in kleine Bündel zerlegt, auf Horden dem Dampf ausgesetzt werden, um eine schnelle und sichere Desinfektionswirkung zu gewährleisten.

Die mit diesem Auseinandernehmen des undesinfizierten Materials betrauten Arbeiter sind in besonders hohem MaBe einer Infeltionsgefahr ausgesetzt, zumal die Lockerung der trockenen Haare mit einer heftigen Staubentwicklung verbunden ist. Es müssen also besondere Vorsichtsmaßregeln getroffen werden, um Infektionen bei dieser Arbeit zu vermeiden. $\mathrm{Zu}$ diesem Zwecke müssen die mit der Arbeit des Auseinandernehmens und der Desinfektion beschäftigten Arbeiter vollständige Desinfektorenanzüge tragen, bestehend aus Mütze, Rock und Hose aus Waschstoff, Schuhen, Mund- und Nasenschutz, etwa in Form der von Kobrak (6) angegebenen Maske. Nach jedesmaligem Gebrauch sind die Anzüge zu desinfizieren. Nach der Beschickung des Desinfektionsapparates haben sich die betreffenden Arbeiter Gesicht, Hände und Onterarme gründlich mit Wasser und Seife zu reinigen, die Hände zweckmäBig mit einer desinfizierenden Flüssigkeit, z. B. einer 5 prozentigen Kresolseifenlösung, abzuwaschen. Der Hauptnachdruck wird dabei auf eine gründliche mechanische Reinigung mit Wasser und. Seife zu legen sein. ${ }^{1}$

Es könnte gegen diese vorgeschlagenen MaBnahmen der Einwand erhoben werden, da nun der Arbeiter, der das Auseinandernehmen der Ballen ror der Desinfektion zu besorgen hat, in ganz besonders hohem Grade der Desinfektion ausgesetzt ist. Dem ist entgegenzuhalten, da $B$ dieser Arbeiter durch die vorgeschlagenen SchutzmaBregeln in sehr weitgehendem Maßae gegen eine Infektion geschützt werden kann, während derselbe bei dem bisherigen Desinfektionsverfahren ebenfalls unter Umständen mit infiziertem Material aber ohne Schutz gegen eine Infektion $\mathrm{zu}$ tun hatte. Ferner ist $z \mathfrak{u}$ bedenken, dab auf diese.Weise der Kreis der gefährdeten Personen sehr verkleinert wird und sich nur auf den Desinfektor beschränkt, während bei einer unsicheren Desinfektion der Haare weit mehr Arbeiter einer Infektionsgefahr ausgesetzt sind.

Eine weitere Verbesserung der bisher geübten SchutzmaBregeln wäre darin zu sehen, daB die Bodenfläche, auf der die undesinfizierten Ballen abgeladen und gelagert werden; aus Zement besteht, um eine gründliche Reinigung zu ermöglichen. Auch der Boden rings um den Desinfektions-

${ }^{1}$ Eine diesen Forderungen entsprechende Anordnung ist von der Fabrikinspektion in Karlsruhe getroffen worden. 
apparat sollte zementiert und letzterer selbst an jeder Schmalseite mit einer Tür versehen sein, um das auch sonst in dem Desinfektionswesen geübte Prinzip der „reinęn und unreinen Seiten" durchführen zu können. Auf diese Weise könnte eine nachträgliche Verunreinigung der desinfizierten Haare mit milzbrandsporenhaltigem Staub und Erde sicher vermieden werden.

Es ist zu erhoffen, daß durch solche Maßnahmen eine sichere Desinfektion der Tierhaare $\mathrm{zu}$ erzielen ist, und somit auch eine erhebliche Abnahme der Milzbranderkrankungen in den RoBhaarspinnereien und ähnlichen Betrieben erreicht wird.

\section{Literatar-Verzeichnis.}

1. Heim, Eine Milzbrandinfektion dnrch Ziegenhaare. Arbeiten a. d. Kaiserl. Gesundheitsamte, 1901, Bd. XVIII. S. 135.

2. Weller, Die gewerblichen Anlagen zur Verarbeitung von Tierhäuten und Tierhaaren vom hygienischen Standpunkte. Vierteljahrsschrift fïr gerichtl. Medizin und äfentl. Sanitätswesen. 1911. Bd. XCI. Suppl.-Heft I. S. 143. S. 498

3. Rembold, Zur Ätiologie des Milzbrandes. Diese Zeitschrift. 1888. Bd. IV.

- Graber, Das österreichische Sanitätswesen. 1896. Nr. 7 u. S.

- Silberschmidt, RoBhaarspinnerei und Milabrandinfektion. Diese Zeitschr. 1896. Bd. XXI. S. 455.

- Russ, Nachweis von Milzbrandbazillen am Pferdehaar. Wiener klin. Wochensehrift. 1907. Bd. XX. S. 663.

- Athanas, Ebenda. 1907. Bd. XX. S. 764.

4. Page, British Industrial Anthrax. The Jowrnal of Hygiene. Vol. IX. p. 357.

5. Reichel, Der Nachweis und die Verbreitung der Milzbrandsporen auf tierischen Ruhstoffen. Bericht über die 5. Tagung der Yreien Vereinigung f. Mikrobiologie in Dresden. 8. bis 10. Juni 1911.

- Centralblatt für Bukteriologie. Bd. L. Beilage zu Abt. I. Ref. S. 83.

6. Kobrak, Respiratoren zum Schutze gegen die Einatmung infektiöser Tröpfchen und Stäubchen. Diese Zeitschrift. 1911. Bd. LXVIII. S. $15 \overline{7}$. 